\title{
Time course of acquired von Willebrand disease associated with two types of continuous-flow left ventricular assist devices: HeartMate II and CircuLite Synergy Pocket Micro-pump
}

Motohiko Goda, MD, a Steven Jacobs, MD, ${ }^{a}$ Filip Rega, MD, PhD, ${ }^{a}$ Kathelijne Peerlinck, MD, $\mathrm{PhD}^{\mathrm{b}}$ Marc Jacquemin, MD, PhD, ${ }^{\mathrm{b}}$ Walter Droogne, $\mathrm{MD},{ }^{,}$Johan Vanhaecke, MD, PhD, ${ }^{c}$ Johan Van Cleemput, MD, PhD, ${ }^{c}$ Klaartje Van den Bossche, MS, ${ }^{a}$ and Bart Meyns, MD,PhD ${ }^{a}$

From the ${ }^{a}$ Departments of Cardiac Surgery; ${ }^{b}$ Molecular and Vascular Biology; and the ${ }^{c}$ Cardiology, University Hospital Leuven, Belgium.

\author{
KEYWORDS: \\ heart-assist devices; \\ heart failure; \\ hemorrhage; \\ transplantation; \\ von Willebrand factor
}

\begin{abstract}
BACKGROUND: Bleeding complications are frequent adverse events in patients supported with axial continuous-flow pumps. Previous retrospective studies demonstrated that bleeding events in patients with the HeartMate II (Thoratec Corp, Pleasanton, CA) were attributed to acquired von Willebrand syndrome. We sought to analyze the von Willebrand factor (VWF) profile in patients receiving a HeartMate II or a CircuLite (Saddle Brook, NJ,) device (Synergy Pocket Micro-pump) prospectively. METHODS: Prospectively analyzed were 34 patients supported with left ventricular assist device (LVAD; 26 with HeartMate II and 8 with CircuLite). The control group comprised 20 patients who underwent heart transplantation (HTx). Blood samples were taken pre-operatively and at 14 days and 3, 6, 9, and 12 months post-operatively.

RESULTS: Patients with LVADs had a high incidence of bleeding complications. From the immediate post-operative phase throughout the entire observation, the VWF ristocetin cofactor activity (Rco)/ antigen $(\mathrm{Ag})$ ratio of patients with HeartMate II and CircuLite devices was consistently lower compared with HTx patients. No correlation was found between the individual VWF:Rco/Ag ratio and bleeding events or transfusion requirements. The VWF:Rco/Ag ratio normalized immediately in patients who received HTx.

CONCLUSIONS: Acquired von Willebrand syndrome was confirmed to occur immediately after the implantation of both types of LVAD and persisted up to 12 months. A lower VWF:Rco/Ag ratio was associated with larger transfusion requirements. Acquired von Willebrand syndrome resolves after LVAD explantation.

J Heart Lung Transplant 2013;32:539-545

(C) 2013 International Society for Heart and Lung Transplantation. All rights reserved.
\end{abstract}

Left ventricular assist devices (LVADs) have played a critical role in therapy strategies for end-stage heart failure patients as a bridge to heart transplantation (HTx) but also as a destination therapy. Recent clinical trials demonstrated the excellent efficacy of circulatory support with

Reprint requests: Klaartje Van den Bossche, MS, University Hospital Leuven, Department of Cardiac Surgery, Herestraat 49, B-3000, Leuven, Belgium. Telephone: +3216344260 . Fax: +3216344616 .

E-mail address: klaartje.vandenbossche@uz.kuleuven.be the HeartMate II (HMII; Thoratec Corp, Pleasanton, CA) and Heartware (Heartware, Inc, Framingham, MA) devices for long-term support. ${ }^{1-7}$ Bleeding complications, including gastrointestinal bleeding during the support time and bleeding risk at the time of HTx, are frequent and possibly life-threatening problems in recipients of these devices. ${ }^{8}$

Meanwhile, a partial left ventricular heart support device, the Synergy Pocket Micro-pump (CircuLite Inc, Saddle Brook, NJ) was introduced. This device could be used in a 
relatively large population of patients with severe heart failure who are not sick enough to justify the aggression of a full VAD implantation. ${ }^{9-11}$ Besides the patients with HMII, we have documented bleeding complications in recipients of the CircuLite device as well.

Previous retrospective studies showed abnormalities of von Willebrand factor (VWF) during HMII support and the return to normal after HTx or HMII explantation, ${ }^{8,12,13}$ a socalled acquired von Willebrand syndrome (AVWS). Other studies reported that AVWS already occurs in the early postHMII implantation phase and persists in the intermediate phase up to 2 months. ${ }^{14,15} \mathrm{We}$ sought to prospectively analyze AVWS for a 1-year period in recipients of VADs and to investigate if patients receiving partial support with the CircuLite device experienced a similar syndrome.

\section{Methods}

This study was approved by the University Hospital Leuven Ethics Committee.

\section{Study design and patient population}

This was a prospective single-center cohort study. The study enrolled 34 consecutive patients ( 27 men and 7 women), with a mean age of $53.6 \pm 15.6$ years (range, 15-74 years), who underwent implantation of an LVAD (26 with HMII and 8 with CircuLite) from January 2010 through June 2011. Indication for HMII implantation was severe heart failure despite maximum medical treatment, including catecholamine support. Indication for CircuLite implantation was having New York Heart Association class IIIb or IV symptoms, despite appropriate medical treatment, but not being inotrope-dependent and ambulatory. ${ }^{10}$ Patient characteristics are summarized in Table 1 . The etiologies of heart failure in HMII patients were ischemic cardiomyopathy in 19, dilated cardiomyopathy in 12 , myocarditis in 2 , and congenital heart disease in 1 . In the CircuLite patients, heart failure was caused by ischemic cardiomyopathy in 4, dilated cardiomyopathy in 3, and a congenital heart disease in 1 . Mean HMII support time was $7.3 \pm 7.4$ months (range, 0.2-43.2 months) and CircuLite support time was $7.9 \pm 8.4$ months (range, 0.2-32 months). Total LVAD observation time was 24 years, and bleeding events are expressed as events per patient-year.

A control set of plasma samples was obtained from 20 patients (16 men, 4 women), aged $43.5 \pm 15.1$ years (range, $14-66$ years), undergoing heart transplantation (HTx) in the same period. Mean follow-up time of the HTX patients was $9.7 \pm 8$ months (range, 4.1-15.4 months).

\section{Platelet inhibition and anticoagulation therapy}

During LVAD support, the patients with the CircuLite device were receiving oral acetylsalicylic acid for platelet inhibition and phenprocoumon as anti-coagulation therapy, with the target prothrombin time-international normalized ratio (PT-INR) of 2.5 to 3.0. Patients supported with the HMII were receiving the same anti-coagulant regimen, but the target PT-INR was lowered to 2.0 to 2.5 . Clopidogrel bisulfate was administered to 7 of 34 LVAD patients (Table 1). Seventeen patients received continuous infusion of heparin, with the target activated partial thromboplastin time (aPTT) of 60 to 80 seconds, instead of oral phenprocoumon for prolonged intensive care. As a routine measure, all patients on assist devices receive continuous oral proton pump inhibitors for gastric protection.

\section{Clinical evaluation and definition}

Baseline clinical data on all enrolled patients, including demographic characteristics, medical history, blood test values, medications, and amount of transfusions were collected and stored by a retrospective record review. Gastrointestinal bleeding is defined as clinically significant in the presence of melena, with decreased hemoglobin level and transfusion required. Epistaxis is defined as a nose bleed requiring a medical intervention.

\section{Blood collection and laboratory analysis}

Prospective blood samples were collected for testing VWF antigen (VWF:Ag), VWF ristocetin cofactor activity (VWF:Rco), and VWF multimers at least once, before LVAD implantation (baseline), at after LVAD implantation at 10 to 14 days, 3, 6, and 9 months, and 1 year. Blood was anticoagulated with $3.2 \%$ sodium citrate and centrifuged at 3,700 rpm for 12 minutes at room temperature. The resulting plasma was frozen in ethanol/ice and stored at $-80^{\circ} \mathrm{C}$ until measurement. Plasma VWF:Ag was measured by automated quantitative enzyme-linked immunosorbent assay (ELISA) with the VIDAS analyzer (bioMériux Corp, Marcy L'Etoile, France). ${ }^{16}$ Normal range of VWF:Ag was $50 \%$ to $160 \%$. As functional analysis of VWF, the VWF:Rco was measured by automated quantitive turbidimetric assay with the BCS XP System (Siemens Healthcare Diagnostic Inc, Tarrytown, NY). The VWF:Rco-toVWF:Ag ratio was calculated, with a ratio $<0.8$ was considered as AVWD. ${ }^{13}$

The multimeric structure analyses of VWF were done as previously described ${ }^{17,18}$ with sodium dodecyl sulfate (SDS)agarose discontinuous gel electrophoresis on a low-resolution $(1.3 \%)$ gel system (LGT agarose type VII; Sigma, Munich, German). The electrophoresis was performed for 16 hours at 55 volts. WVF multimers were transferred to nitrocellulose membranes by electroblotting with transfer buffer $(0.05 \mathrm{~mol} /$ liter phosphates [pH 7.4] with $0.04 \mathrm{~mol} / \mathrm{liter}$ SDS without methanol).

\section{Statistical analysis}

All statistical analyses were performed with Stat View J 5.0 software (SAS Institute Inc, Cary, NC). Differences of baseline characteristics between 2 groups were analyzed by the Student $t$-test. Values for VWF:Ag, VWF:Rco, VWF:RcoVWF:Ag ratio, hemoglobin, hematocrit, and platelets among the 3 groups were compared by the Kruskal-Wallis test. Continuous variables are expressed as mean \pm standard deviation, unless otherwise noted, and the range is presented where appropriate. Categoric variables are expressed as frequencies and percentages, and were compared by Fisher's exact test. The level of statistical significance was set at $p<0.05$.

\section{Results}

\section{Bleeding events}

Patients with LVADs experienced frequent bleeding events, with 37 events in 18 patients. Early and late bleeding in the 
Table 1 Demographic and outcomes data for study cohort

\begin{tabular}{|c|c|c|c|}
\hline Variables $^{a}$ & $\begin{array}{l}\text { HeartMate II } \\
(n=26)\end{array}$ & $\begin{array}{l}\text { Circulite } \\
(n=8)\end{array}$ & $\begin{array}{l}\mathrm{HTx} \\
(n=20)\end{array}$ \\
\hline Age, y & $50.9 \pm 15.2$ & $62.5 \pm 14.3$ & $43.5 \pm 15.1$ \\
\hline \multicolumn{4}{|l|}{ Sex } \\
\hline Male & 20 & 7 & 16 \\
\hline Female & 6 & 1 & 4 \\
\hline Body mass index, $\mathrm{kg} / \mathrm{m}^{2}$ & $23.6 \pm 3.8$ & $22.8 \pm 3.0$ & $25.2 \pm 4.6$ \\
\hline \multicolumn{4}{|l|}{ Etiology of heart failure } \\
\hline Ischemic cardiomyopathy & $15(58)$ & $4(50)$ & $7(35)$ \\
\hline Dilated cardiomyopathy & $9(35)$ & $3(38)$ & $9(45)$ \\
\hline Myocarditis & $2(8)$ & 0 & 0 \\
\hline Congenital & 0 & $1(12)$ & $3(15)$ \\
\hline Post-cardiac surgery & 0 & 0 & $1(5)$ \\
\hline Ejection fraction & $18.3 \pm 7.3$ & $16.6 \pm 4.0$ & $21.8 \pm 11.3$ \\
\hline Atrial fibrillation & $2(8)$ & 0 & $2(10)$ \\
\hline Diabetes mellitus & $3(12)$ & $2(25)$ & $4(20)$ \\
\hline \multicolumn{4}{|l|}{ Oral anti-coagulant therapy } \\
\hline Anti-vitamin $\mathrm{K}$ agents & $24(92)$ & $8(100)$ & 0 \\
\hline Acetylsalicylic acid & $12(46)$ & $8(100)$ & 0 \\
\hline Clopidogrel bisulfate & $3(12)$ & $4(50)$ & 0 \\
\hline Bleeding events (episodes) & $<30$ days $/>30$ days & $<30$ days $/>30$ days & \\
\hline Post-op intrathoracic & 7 (in 5 pts) $/ 0$ & 4 (in 3 pts) $/ 0$ & \\
\hline Gastrointestinal & $0 / 9$ (in 3 pts) & $0 / 3$ (in 2 pts) & \\
\hline Epistaxis & $1 / 0$ & 4 (in 4 pts) $/ 7$ (in 4 pts) & \\
\hline Intracerebral & 2 (in 2 pts) $/ 0$ & $0 / 0$ & \\
\hline
\end{tabular}

HTx, Heart transplantation; pts, patients.

${ }^{a}$ Continuous data are shown as the mean \pm standard deviation and categoric data as number $(\%)$ or as indicated.

CircuLite and HMII groups are indicated in Table 1. In the HMII patients, early bleeding ( $<30$ days after surgery) was predominantly revisions for intrathoracic bleeding (4.06 events per patient-year), intracranial bleeding (1.12 events per patient-year), and epistaxis (0.58 events per patientyear). Late bleeding was gastrointestinal bleeding (0.66 events per patient-year). There was one incidence of thrombosis of a HMII device in a patient after stopping anti-coagulation because of bleeding. In the CircuLite group, only intrathoracic bleedings and epistaxis, both with an incidence of 6.08 events per patient-year, occurred in the early post-operative phase. Late bleeding comprised epistaxis (1.04 events per patient-year) and gastrointestinal bleeding ( 0.44 events per patient-year).

\section{Hemoglobin and platelets among HMII, CircuLite, and HTx patients}

During late follow-up, patient with LVADs were more anemic (at 9 months HTx-HMII: $p=0.03$; at 12 months HTx-CircuLite: $p=0.008$ ) than patients who had a HTx (Figure 1). Platelets levels did not differ significantly among the groups during follow-up (Figure 2).

\section{Von Willebrand profile}

The von Willebrand profile is expressed by the VWF:Ag level, reflecting the total amount of the molecule (Figure 3), the VWF:Rco level, reflecting the thrombocyte binding capacity (Figure 4), and the ratio of both (Figure 5). HMII patients had higher VWF:Ag level $(308.5 \% \pm 93.4 \%)$ preoperatively than CircuLite $(174.8 \% \pm 48.5 \%, p=0.0024)$ and HTX $(169.8 \% \pm 71.1 \%$; $p<0.0001)$. In the immediate post-operative phase, HMII and HTX patients had higher VWF:Ag level $(380.5 \% \pm 120.5 \%$ and $355.3 \% \pm$ $163.4 \%$, respectively; $p=0.0149$ ) than CircuLite patients $(209.1 \% \pm 55.2 \%, p=0.0423)$, a possible reflection of acute-phase response of VWF: $\mathrm{Ag}^{19}$ after invasive surgery. In the post-operative chronic phase, differences among the 3 groups were not significant.

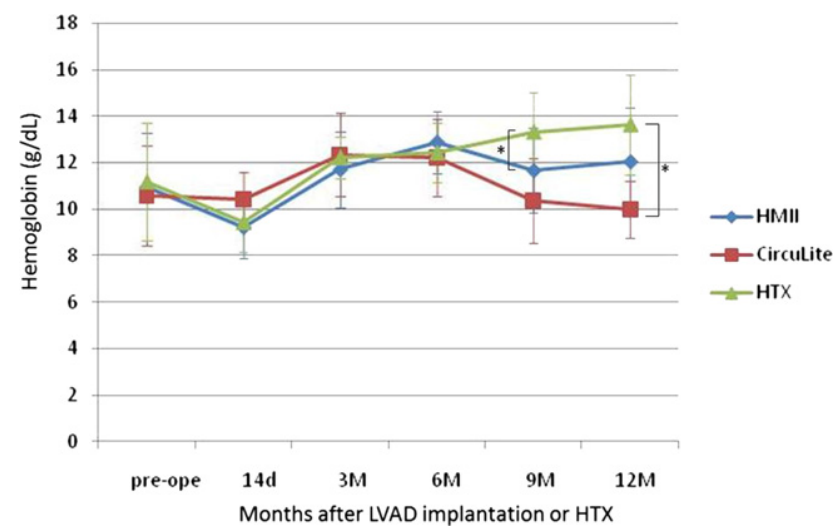

Figure 1 Mean level of hemoglobin is shown in patients undergoing HeartMate II (HMII; $n=26)$, CircuLite $(n=8)$ implantation, or heart transplantation (HTX, $n=20$ ). Patients with left ventricular assist devices (LVADs) are more anemic at longterm follow-up. * Significant difference $(p<0.05)$. Range bars indicate the standard deviation. 


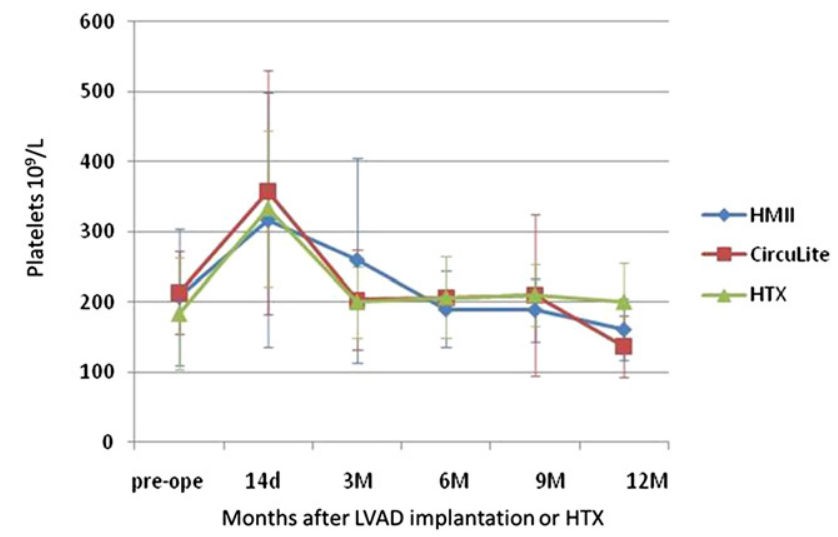

Figure 2 Mean level of platelet counts are shown in patients with HeartMate II (HMII), CircuLite, and heart transplantation (HTX). Platelet counts did not differ significantly among the groups throughout the observation period. Range bars indicate the standard deviation.

HMII patients had higher VWF:Rco level of $317.2 \% \pm$ $129.3 \%$ pre-operatively than CircuLite and HTx patients $(162.1 \% \pm 69.2 \%$ and $109.1 \pm 50 \% ; p=0.0036$ and $p<$ 0.0001 , respectively; Figure 4). In the immediate postoperative phase, HTx patients had higher VWF:Rco level $(362.6 \% \pm 142 \%)$ than HMII and CircuLite patients $(251.5 \% \pm 109.9 \%$ and $112.8 \% \pm 23.7 \% ; p=0.0109$ and $p=0.0002$, respectively). The VWF:Rco of CircuLite patients was also lower than that of HMII patients $(p=$ 0.0244). At 3 months after implantation, HTx patients had higher VWF:Rco level $(218.3 \% \pm 112.7 \%)$ than HMII and CircuLite patients $(131.1 \% \pm 56.9 \%$ and $90.1 \% \pm 35.3 \%$; $p=0.0043$ and $p=0.0028$, respectively). At 6 months after implantation, HTX patients had a higher VWF:Rco level $(171.5 \% \pm 95.7 \%)$ than CircuLite patients $(68 \% \pm 27.7 \%$; $p=0.0166)$. HTX patients had a lower VWF:Rco/VWF:Ag ratio of $0.7 \pm 0.3$ pre-operatively than HMII and CircuLite

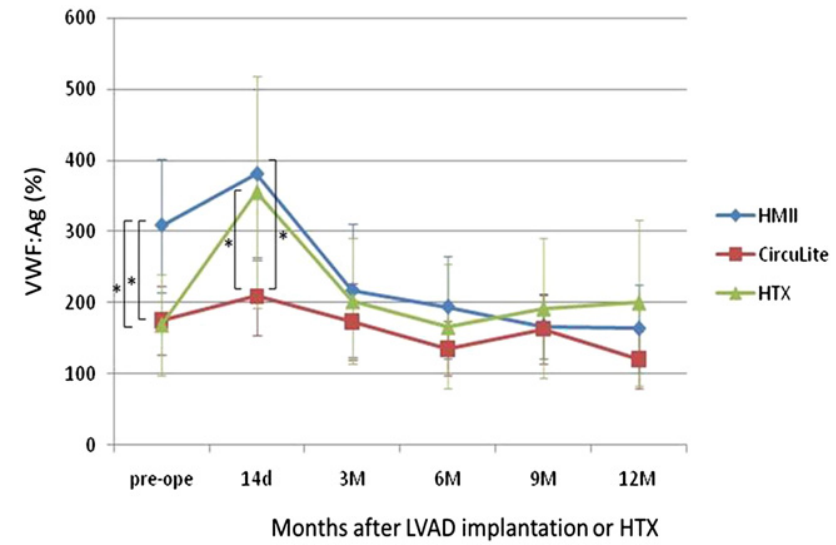

Figure 3 Mean von Willebrand factor antigen (VWF:Ag) levels are shown for the patients with HeartMate II (HMII), CircuLite, and heart transplantation (HTX). HMII patients had higher levels of VWF:Ag pre-operatively than CircuLite and HTX patients. In the acute post-operative phase, HMII and HTX patients had higher VWF:Ag levels than CircuLite patients. *Significant difference $(p<0.05)$. In the post-operative chronic phase, there were no significant differences among the groups. Range bars indicate the standard deviation.

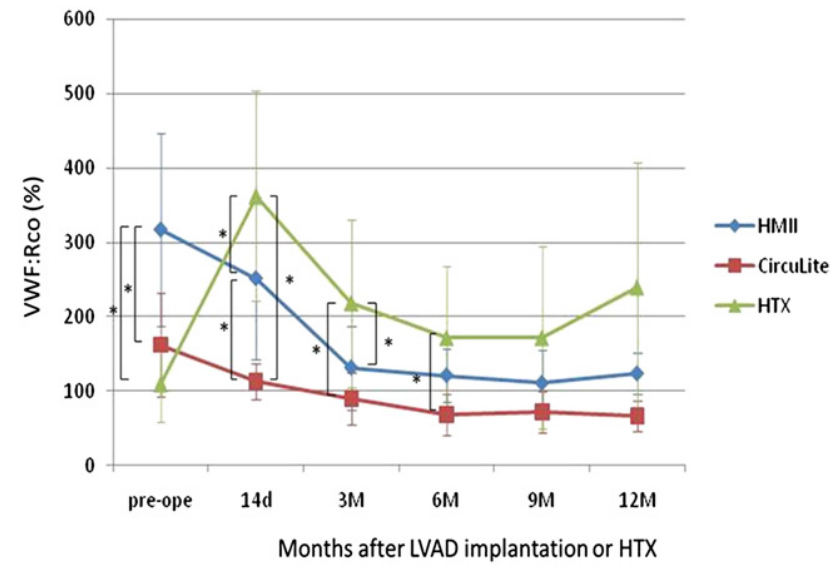

Figure 4 Mean von Willebrand factor ristocetin (VWF:Rco) levels are shown for the patients with HeartMate II (HMII), CircuLite, and heart transplantation (HTX). * Significant differences $(p<0.05)$ in VWF:Rco levels were seen pre-operatively, immediately post-operatively, and at 3 and 6 months after surgery. Range bars indicate the standard deviation.

patients $(1 \% \pm 0.2 \%$ and $0.9 \% \pm 0.2 \% ; p=0.0001$ and $p$ $=0.0449$, respectively). From the immediate post-operative phase throughout the complete observation period, HTX patients had consistently higher VWF:Rco/VWF:Ag ratio than HMII and CircuLite patients.

The relationship between clinical bleeding and the individual level of VWF:Rco/VWF:Ag ratio is unclear. The need for blood transfusion of each patient on support was compared with the mean VWF:Rco/VWF:Ag ratio during the observation period (Figure 6). There seems to be no correlation between both.

Six patients supported with a device (5 HMII, 1 CircuLite) underwent HTx during the observation period, and we obtained samples after HTx in these patients.

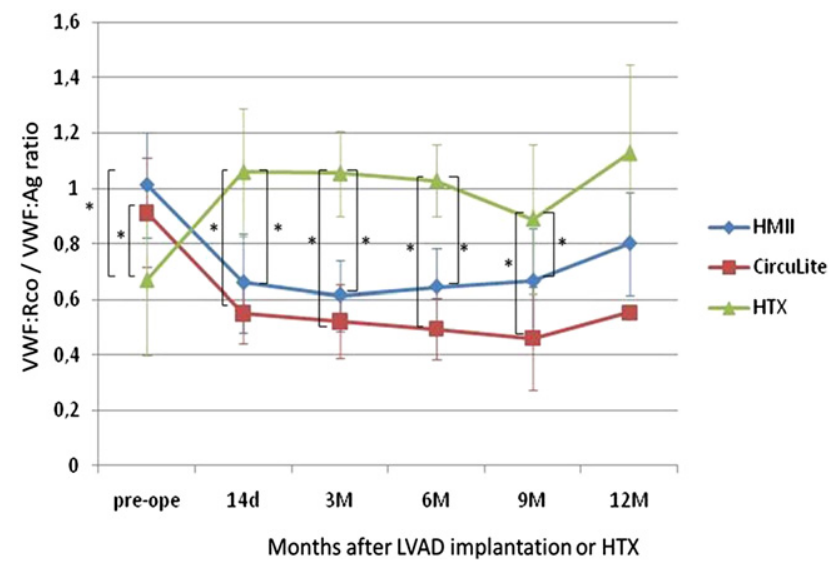

Figure 5 The mean values for the von Willebrand factor (VWF) ristocetin (VWF:Rco)/VWF antigen (VWF:Ag) ratio are shown for the patients with HeartMate II (HMII), CircuLite, and heart transplantation (HTX). HTX patients had a lower VWF:Rco/ VWF:Ag ratio pre-operatively than HMII and CircuLite patients. From the immediate post-operative phase through the observation period after implantation, HTX patients had consistently a higher VWF:Rco/VWF:Ag ratio than HMII and CircuLite patients. *Significant difference $(p<0.05)$. Range bars indicate the standard deviation. 
The VWF:Rco/VWF:Ag ratio increased after HTx and remained normal afterwards (Figure 7).

Electrophoresis of plasma samples from patients supported with HMII or CircuLite for 3, 6, and 9 months were compared with a control patient and a patient with congenital type $2 \mathrm{~A}$ von Willebrand disease (Figure 8). The missing bands in the upper parts of the gel in the HMII and CircuLite patients reflect the loss of high-molecular-weight (HMW) multimers of VWF, similar to the pattern seen in patients with congenital type $2 \mathrm{~A}$ von Willebrand disease.

\section{Discussion}

Long-term LVAD support has resulted in substantial clinical improvement in patients with severe heart failure who were ineligible for HTx. HMII has excellent clinical results ${ }^{1-6}$ and was approved for bridge-to-transplant therapy in 2008 and for destination therapy in 2010 by the U.S. Food and Drug Administration. During support with HMII, the patients require long-term anti-coagulation therapy with acetylsalicylic acid and phenprocoumon to prevent thromboembolic complications. Bleeding complications could be due to anticoagulation therapy but as well to type $2 \mathrm{~A}$ AVWD. ${ }^{8,12-15}$

Type 2A AVWD is characterized by the loss of HMW multimers of VWF. Heyde et $\mathrm{al}^{20}$ reported that aortic stenosis patients could be complicated by high incidence of gastrointestinal bleeding, and this complication is associated with type $2 \mathrm{~A}$ AVWD. ${ }^{21-25} \mathrm{VWF}$ is a plasma glycoprotein synthesized by endothelial cells and megakaryocytes and plays a key-role in the early steps of primary hemostasis and thrombosis. Monomers of VWF $(225 \mathrm{kDa})$ elaborate disulfide-linked multimers (range, 500-20,000 kDa). At sites of vascular injury, multimers of VWF circulating in plasma bind to collagen, swing in blood flow in coiled coil formation, and capture platelets like cobwebs. This phenomenon mediates platelet adhesion and aggregation. ${ }^{24,25}$ The multimers with a higher molecular weight can capture more platelets, like larger cobwebs, and are more effective in adhesion and aggregation. Therefore, loss of HMW multimers impairs the early steps of primary hemostasis and causes bleeding diathesis consequently.

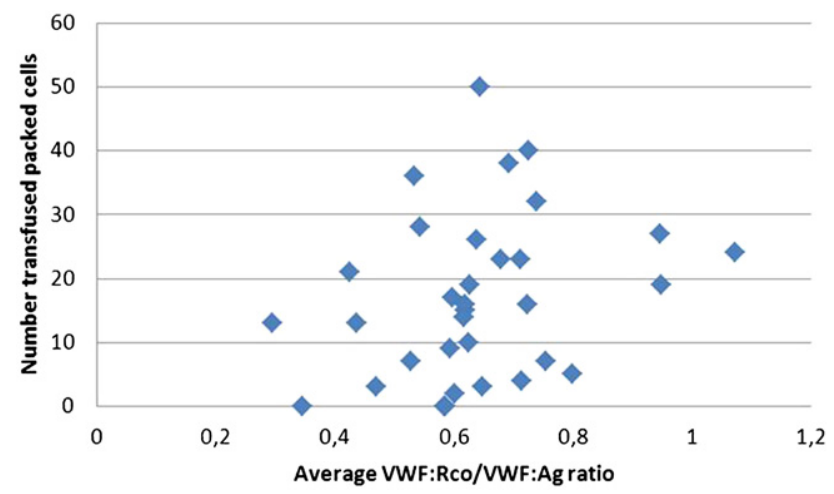

Figure 6 Relationship between need for blood transfusion and average von Willebrand factor (VWF) ristocetin (VWF:Rco)/VWF antigen (VWF:Ag) ratio. There seems to be no significant correlation.

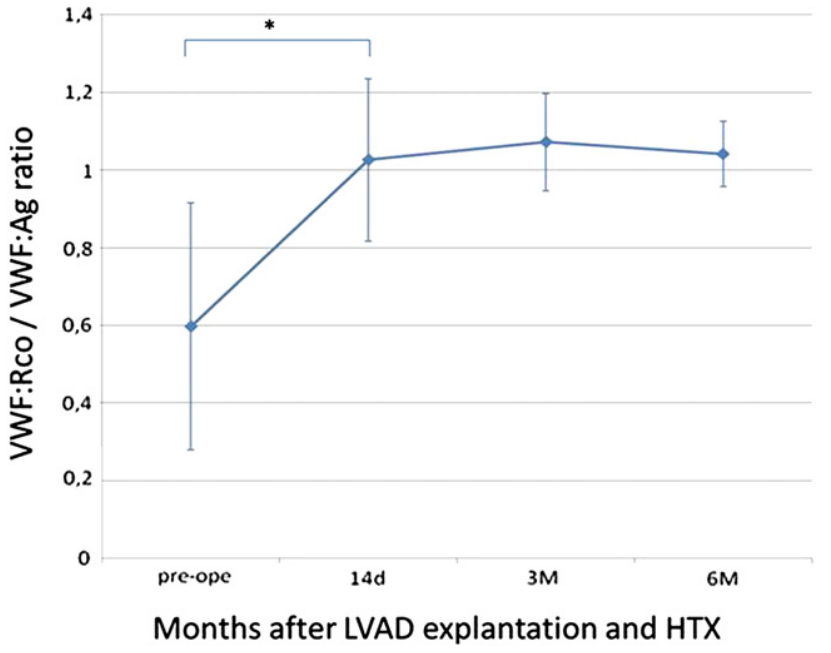

Figure 7 Mean values for the von Willebrand factor (VWF) ristocetin (VWF:Rco)/VWF antigen (VWF:Ag) ratio are shown before and after left ventricular assist device (LVAD) explantation. A significantly difference $\left({ }^{*} p=0.01\right)$ was seen between the preoperative and the immediate post-operative value. In 6 patients who were supported by LVAD (HeartMate II in 5 and CircuLite in 1) before heart transplantation, the VWF:Rco/VWF:Ag ratio normalized after transplantation. Range bars indicate the standard deviation.

Multimers of VWF are decreased under high shear stress according to the following mechanism. In patients with aortic stenosis, for example, blood flow has to go through the constricted aortic orifice. Therefore, velocity is accelerated, turbulences are generated, and shear stress is consequently created. The high shear stress can induce conformational transition of VWF from a coiled conformation to a stretched chain conformation in the direction of the shear stress field. ${ }^{25,26}$ In the stretched VWF multimeric structure, the protease-sensitive bond of VWF is exposed to specific protease activity of the metalloprotease ADAMTS13 (a disintegrin and metalloprotease with thrombospondin motifs, member number 13). ADAMST13 normally regulates VWF function by cleaving VWF and reducing its multimer size. Under high shear stress, the cleavage of ADAMTS13 is also stimulated ${ }^{27-30}$; therefore, the amount of the HMW multimers of VWF is decreased, and type 2A AVWD occurs consequently. This VWF abnormality is improved by valve replacement in aortic stenosis patients but could still persist even after valve replacement if there is patient-prosthesis mismatch. ${ }^{21,22}$ Likewise, the main pathogenesis of bleeding complications in patients with the HMII also has been explained by type 2A AVWD because HMW multimers of VWF were frequently missing. ${ }^{8,12-15}$ We also confirmed that the CircuLite device attributes to AVWD through the same pathogenesis.

Continuous-flow devices create, by the nature of their working mechanism, higher shear stresses than the former pulsatile-assist devices. It is therefore intuitive that all continuous-flow devices might be confronted with this problem. The two continuous-flow devices studied in our series have a different rotor pump system. HMII contains an internal rotor with helical blades that curve around the central shaft, ${ }^{1,2}$ and CircuLite uses a magnetically stabilized 


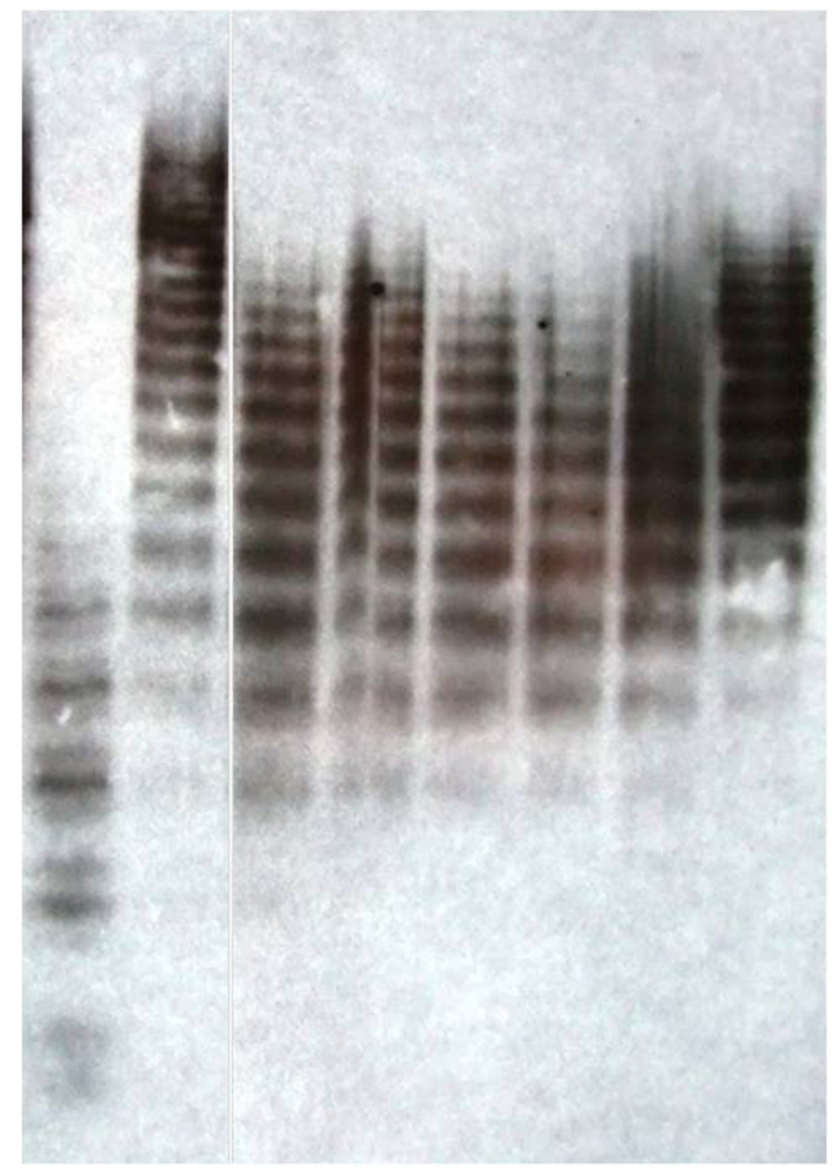

Figure 8 Electrophoresis of the von Willebrand factor multimers, from left to right, for a patient with acquired von Willebrand syndrome, a healthy control patient, a patient with CircuLite at 3 , 6 and 9 months, and patient with the Heart Mate II at 3, 6, and 9 months.

and hydrodynamically levitated rotor with a single-stage impeller. ${ }^{10,11}$ While the rotor spins, the helical blades of HMII and the impeller of CircuLite create the kinetic energy to the blood and continuous blood flow is generated. Through this pumping process, high-speed rotors accelerate velocity and create shear stress. The large multimer of VWF seems sensitive to the mechanical force that is the predominant pathology of type 2A AVWD.

In our cohort, a high incidence of bleeding events was demonstrated in the LVAD patients. Moreover, the observation of the constant lower hemoglobin level of LVAD patients in the post-operative chronic phase than in HTx patients also indicates a possible non-detected state of bleeding. We could confirm these bleeding events were attributed to newly occurring AVWD because the VWF:Rco/VWF:Ag ratio of LVAD patients was constantly lower after LAVD implantation than before. Moreover, the ratio was increased and drastically returned to normal after LVAD explantation. These results support the causal association of HMII and CircuLite with type 2A AVWD. AVWD increased the transfusion requirements not only during HTx in patients with HMII support ${ }^{8}$ but also during long-term follow-up.

Because VWF:Ag is an acute-phase reagent protein, ${ }^{19}$ plasma VWF:Ag level should be normally increased after invasive surgical procedures. Indeed, we found that the VWF:Ag of HTx and HMII patients in the post-operative acute phase was significantly higher than that of CircuLite patients. This might be explained by the fact that HTx and HMII implantation require full sternotomy and extracorporeal circulation, whereas a CircuLite implantation is done through a minithoracotomy without extracorporeal circulation. In contrast, the VWF:Rco/VWF:Ag ratio in HMII and CircuLite patients was already significantly lower than in HTx patients in the immediate post-operative phase. This indicates that the HMW multimers of VWF can be cleaved and AVWD is already developed in immediate postoperative phase after LVAD implantation, which was previously described. ${ }^{14}$

The most frequent adverse event in the late phase among continuous-flow device recipients was bleeding. ${ }^{1}$ It is important to take AVWD into account when a surgical procedure or dental extraction is planned in these fragile patients.

The possibilities to manage bleeding in LVAD patients are limited. It is intuitive to stop the administration of anticoagulants as long as the patient bleeds, and this is the most applied clinical action. It is obvious that it is a challenge to find a balance between the competing risks of bleeding and thrombosis. Once bleeding is stopped, we restart anticoagulant therapy but have adapted the habit to withdraw the anti-aggregation therapy. Besides local bleeding control and transfusion therapy, supplementation of hemostatics could be considered a therapeutic strategy for bleeding events ${ }^{31,32}$ because it is an efficient therapy for AVWD. Plasmapheresis and administration of intravenous immunoglobulins or recombinant factor VIIa are reported to be useful in AVWS based on immunologic pathways and therefore unlikely to be useful in LVAD patients. The administration of desmopressin, VWF-containing concentrates, and anti-fibrinolytics are more likely to exert a positive effect. ${ }^{32}$ Guidelines on this subject are missing and could be helpful.

\section{Disclosure statement}

None of the authors has a financial relationship with a commercial entity that has an interest in the subject of the presented manuscript. Bart Meyns is member of the scientific advisory board of Circulite. This study was partially supported by the framework 7 European Research project SensorArt.

\section{References}

1. Miller LW, Pagani FD, Russel SD, et al. HeartMate II Clinical Investigators. Use of a continuous-flow device in patients awaiting heart transplantation. N Engl J Med 2007;357:885-96.

2. Pagani FD, Miller LW, Russel SD, et al. HeartMate II Investigators. Extended mechanical circulatory support with a continuous-flow rotary left ventricular assist device. J Am Coll Cardiol 2009;54:312-21.

3. Rogers JG, Aaronson KD, Boyle AJ, et al. HeartMate II Investigators. Continuous flow left ventricular assist device improves functional capacity and quality of life of advanced heart failure patients. J Am Coll Cardiol 2010;55:1826-34.

4. Starling RC, Naka Y, Boyle AJ, et al. Result of the post-U. S. Food and Drug Administration-approval study with a continuous flow left ventricular assist device as a bridge to heart transplantation: a 
prospective study using the INTERMACS (Interagency Registry for Mechanically Assisted Circulatory Support). J Am Coll Cardiol 2011;57:1890-8.

5. Slaughter MS, Rogers JG, Milano CA, et al. HeartMate II Investigators. Advanced heart failure treated with continuous-flow left ventricular assist device. N Engl J Med 2009;361:2241-51.

6. Lahpor J, Khaghai A, Hetzer R, et al. European results with a continuous-flow ventricular assist device for advanced heart-failure patients. Eur J Cardiothorac Surg 2010;37:357-61.

7. Aaronson KD, Slaughter MS, Miller LW, et al. Use of intrapericardial continuous-flow centrifugal pump in patients awaiting heart transplantation. Circulation 2012;125:3191-200.

8. Uriel N, Pak SW, Jorde UP, et al. Acquired von Willebrand syndrome after continuous-flow mechanical device support contributes to a high prevalence of bleeding during long-term support and at the time of transplantation. J Am Coll Cardiol 2010;56:1207-13.

9. Meyns B, Ector J, Rega F, et al. First human use of partial left ventricular heart support with the Circulite synergy micro-pump as a bridge to cardiac transplantation. Eur Heart J 2008;29:2582.

10. Meyns B, Klotz S, Simon A, et al. Proof of concept: hemodynamic response to long-term partial ventricular support with the synergy pocket micro-pump. J Am Coll Cardiol 2009;54:79-86.

11. Meyns B, Simon A, Klotz S, et al. Clinical benefits of partial circulatory support in New York Heart Association class IIIB and early class IV patients. Eur J Cardiothorac Surg 2011;39:693-8.

12. Geisen U, Heilmann C, Beyersdorf F, et al. Non-surgical bleeding in patients with ventricular assist device could be explained by acquired von Willebrand disease. Eur J Cardiothorac Surg 2008;33:679-84.

13. Meyer AL, Malehsa D, Bara C, et al. Acquired von Willebrand syndrome in patients with an axial flow left ventricular assist device. Circ Heart Fail 2010;3:675-81.

14. Heilmann C, Geisen U, Beyersdorf F, et al. Acquired von Willebrand syndrome is an early-onset problem in ventricular assist device patients. Eur J Cardiothorac Surg 2011;00:000-000.

15. Crow S, Chen D, Milano C, et al. Acquired von Willebrand syndrome in continuous-flow ventricular assist device recipients. Ann Thorac Surg 2010;90:1263-9.

16. Pittet JL, Barbalat V, Sanvert M, Villard C, Jorieux S, Mazurier C. Evaluation of a new automated ELISA test for von Willebrand factor using two monoclonal antibodies. Blood Coagl Fibrinolysis 1997;8:209-15.

17. Budde U, Schneppenheim R, Eikenboom J, et al. Detailed von Willebrand factor multimer analysis in patients with von Willebrand disease in the European study, molecular and clinical markers for the diagnosis and management of type 1 von Willebrand disease (MCMDM-1VWD). J Thromb Haemost 2008;6:762-71.
18. Ruggeri ZM, Zimmerman TS. The complex multimeric composition of factor VIII/von Willebrand factor. Blood 1981;57:105-8.

19. Lippi G, Franchini M, Targher G, Poli G, Guidi GC. The significance of evaluating conventional inflammatory markers in von Willebrand factor measurement. Clin Chim Acta 2007;381:167-70.

20. Heyde E. Gastrointestinal bleeding in aortic stenosis. N Engl J Med 1958;259:196-200.

21. Sadler JE. Aortic stenosis, von Willebrand factor, and bleeding. N Engl J Med 2003;349:323-5.

22. Vincentelli A, Susen S, Tourneau TL, et al. Acquired von Willebrand syndrome in aortic stenosis. N Engl J Med 2003;349:343-9.

23. Pareti F, Lattuada A, Bressi C, Zanobini M, Sala A, Ruggeri ZM. Proteolysis of von Willebrand factor and shear stress-induced platelet aggregation in patients with aortic valve stenosis. Circulation 2000;102: 1290-5.

24. Di Stasio E, De Cristofaro R. The effect of shear stress on protein conformation. Physical forces operating on biochemical systems: the case of von Willebrand factor. Biophys Chem 2010;153:1-8.

25. Siedlecki CA, Lestini BJ, Kottke-Marchant KK, et al. Shear-dependent changes in the three-dimensional structure of human von Willebrand factor. Blood 1996;88:2939-50.

26. Auton M, Cruz MA, Moake J. Conformational stability and domain unfolding of the von Willebrand factor A domain. J Moi Biol 2007;366:986-1000.

27. Dent JA, Berkowitz SD, Ware J, Kasper CK, Ruggeri ZM. Identification of a cleavage site direction the immunochemical detection of molecular abnormalities in type 2A von Willebrand factor. Proc Natl Acad Sci U S A 1990;87:6306-10.

28. Nishio K, Anderson PJ, Zheng XL, Sadler JE. Binding of platelet glycoprotein $\mathrm{Ib} \alpha$ to von Willebrand factor domain A1 stimulates the cleavage of the adjacent domain A2 by ADAMTS13. Proc Natl Acad Sci U S A 2004;101:10578-83.

29. Gao W, Anderson PJ, Majerus EM, Tuley EA, Sadler JE. Exosite interactions contribute to tension-induced cleavage of von Willebrand factor by the antithrombotic ADAMTS13 metalloprotease. Proc Natl Acad Sci U S A 2006;103:19099-104.

30. Shida Y, Nishio K, Sugimoto K, et al. Functional imaging of sheardependent activity of ADAMTS13 in regulating mural thrombus growth under whole blood flow conditions. Blood 2008;111:1295-8.

31. Cushing M, Kawaguchi K, Friedman KD, Mark T. Factor VIII/von Willebrand factor concentrate therapy for ventricular assist deviceassociated acquired von Willebrand disease. Transfusion 2012;52:1537-41.

32. Tiede A, Rand JH, Budde U, Ganser A, Federici AB. How I treat the acquired von Willebrand syndrome. Blood 2011;117:677-85. 